ETHICAL ISSUES IN RESUSCITATION

\title{
Futility, appropriate care, and orders not to resuscitate: Who makes decisions and how?
}

\section{Christopher D. Lewis and Michael S. Ewer}

The University of Texas, Houston Texas, United States of America

\author{
Address for correspondence: \\ Michael S. Ewer \\ Box 43 \\ The University of Texas \\ M. D. Anderson Cancer Centre \\ Houston Texas \\ 77030
}

United States of America

Email:

mewer@mdanderson.org

\section{INTRODUCTION}

"Resuscitation" derives from Latin roots meaning to reawaken or set in motion again. Resuscitation implies reanimation of a body seemingly bereft of life. Resuscitation, as the authors use the term, is an overt effort to thwart apparently imminent death.

Early resuscitation techniques targeted accident victims harmed by trauma or drowning and sought to restore air movement into the lungs through mechanical chest movement. Attempts dating back to the mid 18th century have been reported. As insight into the pathophysiology of cardiac arrest improved, the procedures to resuscitate or re-animate expanded to include mouth-to-mouth inflation of the lungs and cardiac compression, not only closed, but also even direct squeezing of the heart in an opened chest.(I)

Early resuscitation raised little ethical concern. Drowning victims were often youths without underlying pathology and attempts to prolong life were presumed to be desirable and appropriate. As resuscitation techniques improved its application expanded to potentially include anyone suffering cardiac arrest - raising the risk that patients might be resuscitated despite painful irreversible conditions likely to cause imminent death regardless. Resuscitation's

\section{ABSTRACT}

Ethical issues in resuscitation arose once life-prolonging interventions advanced to the point where short-term cardiac resuscitation became plausible in patients in cases where imminent death was irreversible. The authors argue that ethical dilemmas arise from disputes over continued treatment when stakeholders either disagree about the meaning of appropriate care as a result of differing beliefs on the meaning of an acceptable outcome and/or the extent of a treatment's probable efficacy. The authors conclude that even though communication and transparency can help prevent these ethical dilemmas, unavoidable conflict over proper interventions should be resolved through a decision-making process grounded in both medical reality and the principles of patient self-determination. Thoughtful regulatory guidance can aid the understanding of rights and responsibilities when the desirability, efficacy, and medical indication of life-prolonging interventions are in dispute. The authors outline such a process. The authors suggest that seeking clear regulation in this arena is a worthwhile ethical and practical objective for physicians to reduce both the likelihood of conflicts and the burden of unavoidable conflicts despite transparency and communication.

SAHeart 2010; 7:272-279

indications became less clear, both to patients' families and their physicians. Social, ethical, and legal problems consequently developed, and this paper discusses an appropriate framework for decision-making algorithms for resuscitation.

\section{PERMISSIONTOTREAT}

In the English legal tradition offensive contact is considered battery unless the ostensible victim consents. ${ }^{(2)}$ Even non-controversial interventions such as an appendectomy to remedy acute appendicitis have side-effects which offensiveness to the patient would invite legal treatment as battery, absent effective consent. A patient who is awake and able to consider a treatment's risks and benefits - the case for most care-seeking patients - presents a straightforward opportunity to accomplish the ethically crucial task of obtaining effective consent. However, a patient able to give consent, 
who on learning about available options refuses proposed treatments, has the privilege to avoid the proposed offensive contact.

Candidates for cardio-pulmonary resuscitation (CPR) with no pulse and who are not breathing cannot articulate consent. Reasonable people might disagree whether a certain patient would under the circumstances consent to resuscitation. Since broken ribs, aspiration of vomit and infectious illness are all foreseeable consequences of CPR, such contact seems ripe to be considered treatment as battery without consent. Since CPR candidates cannot express consent at the time treatment is offered, how does one deteremine if such treatment is ethically possible?

In emergencies - both for the incompetent and in other circumstances - legal fictions are indulged to support treatment without a patient's express consent. ${ }^{(3)}$ Parents may give "consent" for a patient legally deemed too young, and an unconscious patient may be presumed to give consent to emergency treatment. In resuscitation, ethical problems arise because no one can know what decision a patient would have made in the face of the medical developments that occurred after the moment the treating physicians had last given instructions to his physicians. Stakeholders can reasonably disagree on the patient's decision. The more a patient's condition suggests that intervention could lead to an acceptable recovery, the clearer the case for presumed consent. Where non-treatment is thought to inevitably lead to death the case for presumed consent appears strong. Indeed, some question how one might ethically avoid presuming consent for a potential CPR candidate.

Stakeholders' miscommunication, misunderstanding and unavoidable differences in perspective can lead to disagreements in either direction: Families may expect treatments that physicians doubt a patient would choose, while physicians may advocate interventions families believe would be contrary to a patient's preferences. Physicians and patients can seek judicial intervention to require or forbid treatment over others stakeholders' objections. Judicial determination is burdensome: Costly legal disputes pit families against treating professionals yet cannot determine the treatment that a patient would have opted for. Confrontation - even in the supposedly civil context of a legal proceeding - provides poor outcomes even for prevailing parties. Better mechanisms are however warranted in the high-stakes, high-cost decision to resuscitate where a patient unlikely to survive regardless of intervention should be resuscitated.

\section{RESUSCITATION: IS EVERYONE AT RISK?}

The increasing availability of resuscitation means people whose natural transition from life to death include a cardiac arrest could likely get resuscitated if medical intervention is on hand. The availability of tools such as ventilators increases the potential for non-therapeutic intervention via long-term intensive care for a patient without a plausible chance to recover his/her decisionmaking capacity; interact with family; or indicate the need of selfdetermination or individuality by, for example, expressing a preference for solitude or for particular company.

Resuscitation does not necessarily benefit everyone with cardiac arrest nor was it developed merely to change the moment of declared death at the end of a patient's life.

A mechanism needs to exist to ensure that non-resuscitation is available as an ethical alternative to presumed consent. Since death is the alternative many places have implemented institutional policies to resuscitate in the absence of contrary instruction where resuscitation appears to be the standard of care - to either avoid liability - or by assuming that most patients will benefit from resuscitation.

\section{THE “DO NOT RESUSCITATE” (DNR) ORDER: NOT A COMPLETE SOLUTION}

One solution could be in the form of a patient's advance notice in terms of treatment limits. A medical order, placed in the medical record and made known to all treating professionals, could then bar resuscitation under circumstances beyond the limit. Consent can also be obtained from a proxy legally authorised to make medical decisions for the patient. In some jurisdictions, statutes authorise physicians to consider a patient's written medical directives - or a properly designated proxy's consent - as equivalent to a conscious patient's own decision. Proxies have practical strength: They can react to developments while the patient is unable to, and exercise discretion in circumstances which could never be considered by the patient. 
These advances in consent resulted from the ethical ideal of patient autonomy and self-determination. However, the focus on obtaining consent resulted from the dubious assumption that patients and their decision-makers would act rationally. When faced with situational stress, some loved ones aggrieved by a relative's unexpectedly worsening condition entertain unreasonable expectations about continued intervention. Some patients may fear a medical practitioner's knowledge of a DNR order and refuse to express consent to any treatment limits fearing that a DNR order would undermine the physicians' zealousness prior to arrest. Some patients solve this concern by appointing a proxy, who can withhold from physicians the consent's limits until the knowledge is no longer worrisome. Using proxies invites conflict between proxies and patients' own statements limiting consent.(4,5) Despite the risks posed by proxies, proxies' statements regarding patient preferences may be more accurate than those of treating physicians, making proxies superior in realising the ethical ideal underpinning patient consent. ${ }^{(6)}$

\section{BOUNDED RATIONALITY AND UNAVOIDABLE DIFFERENCES}

What has not disappeared is recurring friction between stakeholders over the appropriateness of life-extending interventions to medically complex patients. Adding to the conflict are: Imperfect communication on realistic medical probabilities; misunderstanding of the patient's objectives and preferences; and the difficulty of an appointed proxy to make decisions while a loved one's recovery prospects are deteriorating.

The optimal solution is not to absolve one side of responsibility, however simple the solution might be. Decision algorithms that would pit patients' advocates against treatment teams does not improve communication between treating physicians and those seeking their advice. Innovative provider to patient communication is needed to help align the understanding of all parties so we can better realise our ideal of self-determination and informed consent.

Unfortunately, innovations such as improved access to patient advocates and counselling personnel compete for the very resources theoretically available to provide treatment. Different localities that prioritise resources differently can be expected to make different decisions regarding the advancement of different aspects of the entire treatment process, including not only access to interventions but also improved access to decision-making support. However, resource limitations are a global universal. Even if sufficient investment of training, personnel and other resources could eventually bring every patient's decision-makers into agreement with some treatment team willing to perform the desired interventions, this optimal solution would come at the expense of other priorities. ${ }^{(7)}$ Decision-making theory and the limits of rationality in a world with constrained resources combine to ensure that stakeholder agreement regarding appropriate treatment will remain imperfect.

The authors do not denigrate prioritising the ethical value of selfdetermination by elevating patient autonomy through innovative communication. The authors rather suggests that - despite any community's absolute measure of available resources - each will observe a non-zero incidence of failure to afford every patient a team of medical professionals whose treatment plans can be universally aligned with both the preferences of the patient's informed decision-makers and the limitations of available treatment resources. Therefore, the authors urge planning - not ideal planning, but adequate planning - to manage potential conflicts in order to reduce the negative impact on patients, medical professionals and communities.

The authors thus assume the inability to obtain effective consent to withhold treatment under the circumstances of some individual patients. Where consent to withhold treatment cannot be obtained or is revoked, a solution is needed. An ethical dilemma will occur especially when scarce or costly therapy resulting in resource shortages impact patients with a clearer opportunity to benefit from intervention: Does one abandon patients and the principle of their self-determination; or does one accept ethically unacceptable risks or wasted resources? Medical professionals and patients' families need a reliable mechanism to resolve, with modest overhead, unavoidable disputes over the inappropriateness of treatment. Providers should not be compelled to provide indefinite maintenance for patients for whom medical opinion holds recovery implausible and families should be aware of their alternatives when the alignment of their goals and those of their treatment professionals cannot be reconciled. 


\section{DECISIONS, UNCERTAINTY AND MEDICAL}

\section{REALITY}

Outcomes estimates are limited by the information available at the time and the certainty that conditions change. The likelihood of meaningful survival can change rapidly. When patients come to physicians and hospitals, they expect benefit. However, interventions can fail. The patient with serious ischaemic heart disease advised to undergo revascularisation surgery, but who does not survive, might have had a longer life without intervention. The inability to predict negative outcomes often results in anger and a sense of betrayal towards those who proposed or conducted an intervention.

The Hippocratic Oath famously admonishes physicians first to do no harm, then to advocate the interest of each patient. Modern medical practice - complete with organ waiting lists; blood products shortages; costly experimental therapies with unknown outcomes or adverse events profiles; and established but competing therapies with different outcomes and adverse events profiles - makes it difficult to ascertain what course would constitute the avoidance of harm, and sometimes even whom one might harm. Ascertaining a patient's preference regarding resuscitation can be challenging, considering the medical conditions and likely outcomes, particularly when the patient's condition is unlike any the patient ever imagined. Assuming the patient desired "everything" be done to stave off death, even for an hour of unconsciousness, that course of action could easily interfere with meaningful treatments of other patients to whom an equal duty is owed, and whose prognosis may justify prioritised resource allocation.

The micro-ethical world in which a physician can properly look only to a single patient in deciding the ethical course of action is a world that does not exist today and may never have. The world in which physicians treat patients routinely requires competing interests to be weighed in connection with scarce therapeutic agents and limited institutional resources. In the real world, all stakeholders face hard choices regarding the risks they will accept and the care that can be ethically offered. This world simply does not allow physicians to absolve themselves of ethical hardship by making available every possible resource for every patient until resuscitation has clearly failed. If we tried, our institutions and our society could not afford it.
Someone must allocate resources, including physicians' limited time, in accordance with some standard akin to a mass-casualty triage environment. Incautious dispensation of scarce resources betrays more than the patients who might be restored to health: It betrays the community whose resources are expended to obtain scant result due to wasteful resource allocation decisions. Impassioned pleas for treatment may be easier to accept, but someone must advocate for those who are absent: Those needing treatment after an overrun personnel budget prevented sufficient treatment personnel; those whose outcomes turn on the availability of one more unit of blood; and those whose taxes and insurance premiums are resources in the hands of a fiduciary expected to improve health outcomes. Patients likely capable of benefit should be hastened toward likely-effective interventions, yet some patients can be effectively aided only to mitigate discomfort. Misallocating resources by oversupply is as unethical when it offers no aid to the recipient because the treatment is futile, as it is unethical when a patient has expressly refused the treatment and its provision is an assault.

Even in this world of uncertain outcomes, an optimal course of treatment may be ascertainable for a patient. Yet, the mass-casualty environment of modern medical institutions makes it possible that dedicating resources to provide optimal treatment for some may jeopardise an institution's outcomes for other patients. ${ }^{\left({ }^{8}\right)}$ The capacity to benefit is a significant factor in evaluating the ethics of allocating resources among existing and potential patients. ${ }^{(9)}$ Futility represents one extreme range of capacity to benefit and provides a useful place to consider resolution of resource allocation conflict.

While we must strive to offer the best possible care to every patient, our recommended interventions must remain flexible, and vary not only with patients' predicted outcomes but with the available resources at the time and place of treatment. The certainty with which physicians recommend an intervention must relate to the likelihood of the outcome. Because outcomes are uncertain, recommendations should overwhelmingly be made with the understanding that risks are ultimately borne by the patient.

It is imperative that full information on recommended alternatives be provided. There is a cost in time and training to enabling patients' informed consent, but failure to meet this standard 
departs from society's expectations, thus necessitating this outlay. Failure to keep decision-makers adequately informed undermines the principle of patient self-determination and individual autonomy Purported consent obtained by limiting the options and risks disclosed to decision-makers is not informed consent, and is merely illusory.

\section{MEDICAL UNCERTAINTY AND THE DECISION CONTRIBUTION CHART}

Medical probability lies within trained professionals' expertise, but weighing risks and the value of outcomes is the realm in which informed consent was intended to govern. Where options are few and likely outcomes are reasonably certain, independent medical judgement might invariably lead to the same proposed intervention. In situations like these, especially when time is of the essence, the treatment decisions are, within reason, primarily made by the treating physicians. For example: Relatives bring an otherwise healthy youth with profusely bleeding penetrating trauma to the throat to an emergency department. An independent panel of hypothetical experts is required to unanimously agree that surgical repair is the only option. What is more, panic-stricken relatives need to give consent prior to treatment: Given the time span, this exercise could, in all likelihood, result in death.

Figure I depicts a triangular graph in which a hypothetical independent expert panel's fraction of assent to a proposed course of treatment appears on the horizontal axis labelled the "Index of appropriateness". (10) As the expert panel result approaches 100\% in terms of agreement, the height of the graph approaches zero. To the left zero height indicates zero patient contribution to decision-making. If treatment is desired at all, there is but one result. To the right, a zero triangle height indicates a treatmentteam contribution to the decision of $100 \%$. When there is no medical uncertainty on the appropriate treatment, the decision results in the patient being treated. In an informed-consent driven treatment environment, patients and their consent-giving representatives should be kept as informed as possible even when their input is less crucial, so that once required they can make the most informed decision. The parties involved should be emotionally prepared to consider the patient's developing medical condition when a choice arises. There could also be times when the treatment team need to keep the patient's decision-maker informed, but with little reason to solicit input other than perhaps to withdraw consent.

However, as treatment decisions are made in the face of unknowns rather than the basis of medically ascertainable certainties, patients and those expressing their consent should increasingly dominate decision-making.

For example: A patient with prostate cancer opts for treatment due to the condition's aggressiveness. Moreover the co-morbidities of surgery and radiotherapy carry similar risks and benefits. In this case, a hypothetical panel of experts is unlikely to reach unanimous consensus, as the various merits of the different therapies plausibly appeal to different professionals who each have a respectable basis of opinion. If this hypothetical panel of medical experts is evenly split, with half favouring a given option, there is no medical preference in the selection of treatment; the decision lies solely with the patient. Figure I indicates this: Where the expert panel's agreement is exactly $50 \%$, the vertical line drawn through the graph intersects the graph at its apex, and the consent-giver's contribution to the decision is absolute and the

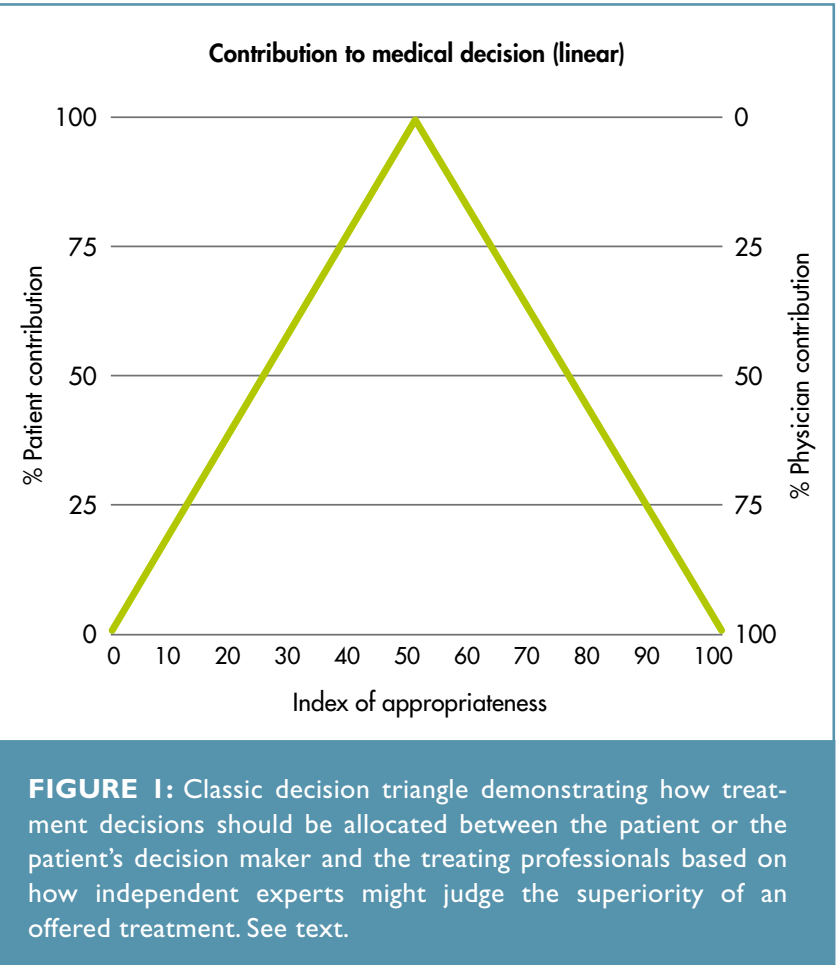




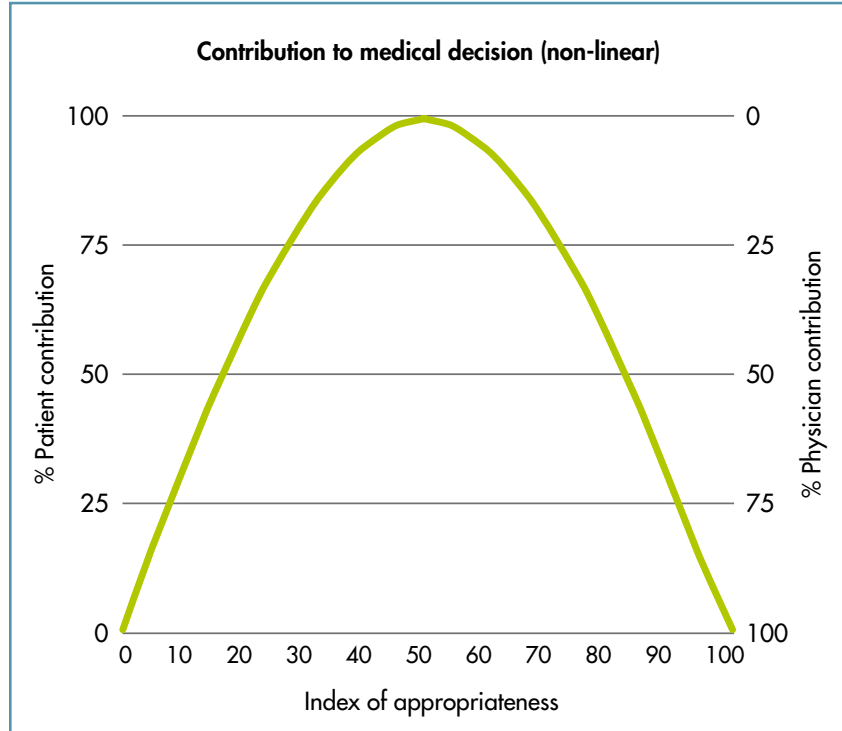

FIGURE 2: The decision triangle makes the assumption that the decision-making process is linear; yet, as appropriateness of alternatives becomes subject to judgements regarding the value of outcomes and the significance of probabilities, the ethical principle of patient self-determination requires great deference to be accorded the preferences of the patient. Accordingly, the sinusoidal pattern reflects an improved algorithm which reflecs the distribution of decisions among the stakeholders, more quickly accelerating to patient advocates the fractional contribution to medical decisions as a case's prospects diverge from apparent medical certainty. See text.

contribution of the medical team is zero. This does not mean the medical team did not provide information to the patient or the patient's decision-makers, but that the physician's role is informative and not persuasive. If there is no basis on which to advocate any particular therapy, there is every reason to solicit the patient's most informed decision in selecting treatment. Uninformed consent is contrary to the patient self-determination, so the need to provide accurate information regarding the choices is very high despite the possible equivalence of the treatments.

How to value risks and uncertainties is not plainly established in the ethics literature. The correct and ethical result that should be reached at the extremes - deadlocked independent experts or absolute unanimity - seem clear in the light of competing interests, but such circumstances may not characterise many disputed medical decisions. As one drifts from the clear poles of certainty, the weighing of competing outcomes' value to particular patients may drive decision-making into the hands of patients in a nonlinear fashion.
Figure 2 presents a graph of contribution to medical decisions but depicts greater patient contribution with slighter departure from apparent medical certainty. Whether medical probability favours approaching patients as an advocate for a particular course of treatment or approaching them to dispense information from which to obtain a decision without preference by physicians, it is important to keep patients and their decision-makers informed so that they will be as prepared as possible to make appropriate decisions as conditions change.

\section{NON-REGULATION OF DISPUTES OVER MEDICAL FUTILITY DETERMINATIONS ENABLES CONFLICTS OF INTEREST AND INEFFICIENCY}

Poorly-regulated disputes over the proper course of treatment lead to adverse consequences. Physicians can fear adverse consequences from disputes over patients' treatments: The incentive to placate family members in the event of a dispute can undermine the legitimate concern to save scarce resources for patients not yet present and able to complain. Opposing desires of patients and proxies on one hand and physicians and hospital administrators on the other create a problem beyond the ethics of resource allocation. Stakeholders should be working together with congruent interest to maximise useful and meaningful life, but opposing views on treatment can position patients and physicians as adversaries. Under such circumstances, patient care can be frustrating. Once the physician-patient relationship becomes adversarial, liability fears add significant conflict of interest. The possibility that litigation, employment prospects, relationships with medical institutions, professional grievances, and other sequelae of patient dissatisfaction could impact a clinician's career when the stress of disagreements and conflict of interest arising when clinicians find themselves at odds with patients, exacerbates stress.

Exacerbating the difficulty of analysing disputes over resuscitation is the imprecise terminology framing the disputes. Futility is typically asserted as an absolute. In reality a treatment can only be termed futile once intervention was given in full measure until the patient was so obviously beyond help that further resuscitation was agreed to be wasteful by all witnesses and that continued intervention was considered to have no desirable effect. To so demonstrate 
futility is undesirable. Futility analysis is also complicated by the rare cases in which recovery occurs despite physicians' conviction of irreversibility. The limit of medical knowledge prevents knowing, with absolute certainty, that continued treatment of a live patient will be futile. (II)

The principle of personal sovereignty prevents a system in which patients, once delivered into the hands of medical professionals, must accept whatever care the physicians choose to provide. The fact that every patient is different, and that patients have legitimately different interests and personal priorities, makes it impossible to dictate in advance the proper care for every diagnosis. Yet, patients cannot be permitted to demand any treatment regardless of potential benefit or resource limitations. All stakeholders need an established mechanism by which conflicts regarding appropriate care can be resolved without necessarily invoking a judicial system whose expense and adversarial nature work against the interests of all parties.

\section{REGULATION OF DISPUTED FUTILITY}

\section{DETERMINATIONS}

Litigating the appropriate care to give patients following disputes is potentially costly both in funds and in time. A different mechanism is needed: One where medically inadvisable treatment may be withdrawn without liability for abandonment where it should be made sufficiently clear that there is little chance of misunderstanding of the parties' rights as the procedure is followed. The patient's interest in finding desirable alternatives must be balanced against the need of providers and the community not to be drawn into indefinitely expending resources without benefit. If the rights of the parties are sufficiently clear, professionals and their institutions can be protected from adverse consequences of exercising medical judgment even in the most antagonistic terminations of treatment. The precise balance of these interests must necessarily depend on each jurisdiction's differing people's needs and expectations. The rules should be sufficient to prevent disaster and tolerable to enough people that it can be accepted and implemented without engendering a public sense of injustice.
Some jurisdictions authorise physicians to withhold life-sustaining treatment they deem futile once certain conditions are met to protect the interests of other stakeholders. Texas, for example, enacted a statute to create a limited period in which life-sustaining care must be continued following a hospital's review of the treatment team's determination of futility. During that time, treatment advocates may seek a facility willing to provide the desired care. There is a cost to this delay, but the delay is not indefinite as the duty lasts but ten days. After the prescribed period - with notice and an opportunity to find alternate caregivers - treatment may be terminated without liability.

This particular balance may not fit every community, which may demand less or offer more. A different balance must be reached suitable to local sensitivities. However, some clear rule establishing the circumstances under which withdrawal of intervention will be protected is needed to prevent other ethical problems marring the treatment decisions of physicians, such as: Conflicts of interest arising from fear of reprisal through grievances or malpractice suits (which sufficiently clear regulation would reduce to empty threats); efforts to deceive decision-makers about the state of a patient's health in order to manipulate their decisions; or other potential ethical disasters. Clear rules make ethical conduct safer and prevent confusion among stakeholders.

\section{INVOKING FUTILITY POLICIES}

Announcing reliance on a statute protecting withdrawal of treatment is not the ideal mechanism through which a treatment team informs a patient's family that the end is near. The broader relationship of an institution to its stakeholders militates against reliance on futility policies when alternatives exist.

- Firstly, efforts to improve transparency in communication with patients and their decision-makers can be effective to prevent such conflict; and

Secondly, invoking a futility policy may itself do more harm than good: If a patient's prognosis does not suggest more than the briefest survival regardless of intervention, there may be little 
to be gained by antagonising a family with what will likely be perceived as abandonment.

The particulars of local futility laws will offer guidance in this regard. Under Texan law, for example, a 3-day survival prognosis should prevent invoking a futility policy because the institution would have ceased providing intervention with the expiration of the patient before the statute's benefit ripened. Thoughtfulness and sensitivity must govern. Futility policies that call for cessation of interventions over the strenuous objections of family members are no reason not to work to avoid discord with families and they leave room to accommodate families when the gain to the institution and its other stakeholders is too minimal to justify the conflict and cost associated with the futility policy. As with other medical tools, judgement is paramount.

\section{CONCLUSION}

Medical professionals have an important role in leading patients and their decision-makers to appropriate treatment decisions. Physicians have a proper role as an advocate for interventions offering clear benefits, but should offer disinterested but informative counsel when no particular treatment is clearly superior. Patients know that modern medicine offers outcomes that were difficult to imagine a century ago, yet disagreements over plausible outcomes and the appropriateness of interventions will continue.

Despite improved methods to keep patients and their proxies adequately informed to enable them to make decisions, some disagreement over emotionally-charged decisions surrounding the appropriateness of resuscitation remains unavoidable. Regulation to define parties' rights in the event of disagreement on appropriate care helps providers to provide unbiased, honest, and ethical medical judgment. Definite responsibilities in the event of disputes can be defined without dictating medical practice or undermining advances in end-of-life treatment. By enhancing the security of professionals in discharging their ethical duty by offering their honest appraisals and seeking to keep patients fully informed, regulation can aid patients and physicians without restraining either patients or physicians in the range of treatment.
Regulation should avoid micro-managing the physician-patient relationship, and encourage patients to access like-minded professionals instead of seeking indefinitely to maintain an unhappy and confrontational status quo through fear or litigation. Because clarification of rights offers both a mechanism to manage the parties' expectations and a shield for ethical conduct, it is the authors' view that physicians should advocate specific regulations to balance the interests of the stakeholders in the face of disputes over resuscitation. Where treatment teams' efforts to keep patients' decision-makers informed fail to maintain their support for a course of action consistent with sound medical judgement, reasonable regulation should enable providers to restrict care to be consistent with systems such as the decision curves presented here.

\section{REFERENCES}

I. Ewer M, Ali M, Frazier OH. Open Chest Resuscitation for cardiopulmonary arrest related to mechanical impairment of circulation: A report of two cases. Critical Care Medicine 1982; 10:198-9.

2. Sfikas PM. Informed consent: How performing a less invasive procedure let to claim of battery. J Am Dent Assoc 2006; 1 37:722.

3. Mendelson D. Substituted consent: from lunatics to corpses. J Law Med 2007; 14:449-62.

4. Altman LK. The man on the table devised the surgery. New York Times, 2006 Dec. 31 .

5. Ewer MS, Taubert JK. Advance directives in the intensive care unit of a tertiary cancer centre. Cancer 1995:76:1268-74.

6. Shalowitz DI, Garrett-Mayer E, Wendler D. How should treatment decisions be made for incapacitated patients, and why? PLoS Med 2007;4:e35.

7. Simon HA. Models of bounded rationality, Vol.I: Economic analysis and public policy, MIT Press 1982.

8. Pauls M, Leblanc C, Campbell S. Ethics in the trenches: Preparing for ethical challenges in the emergency department. CJEM 2002;4:45-8.

9. Maddox PJ. Administrative ethics and the allocation of scarce resources. Online J Issues Nurs 1998;3:ms5.

10. Ewer MS. Philosophy of care, decision-making and ethical considerations. Crit Care Clin 1989;5:679-95.

1।. Ewer M. The definition of medical futility: Are we trying to define the wrong word? Heart \& Lung 2001;30:3-4. 